\title{
Uptake and effectiveness of the Australian telephone Quitline service in the context of a mass media campaign
}

\author{
CL Miller, M Wakefield, L Roberts
}

Tobacco Control 2003;12(Suppl II):ii53-ii58

See end of article for authors' affiliations

For correspondence: C L Miller, Tobacco Control Research and Evaluation, The Cancer Council South Australia, PO Box 929, Unley, South Australia 5061, Australia; cmiller@cancersa.org.au

\begin{abstract}
Objectives: Since June 1997, Australia has run its first nationally coordinated mass media anti-smoking campaign, with all States collaborating to offer a standard Quitline service. An overview of the Australian national Quitline service is presented as well as two studies describing (a) the relationship between television advertising and call volume and type, and (b) the quit rates of callers over time. Design: Data on extent of advertising, as measured by weekly television target audience rating points (TARPs), is compared with weekly call volume and disposition. A randomly selected sample of callers was followed up at 3 weeks, 6 months and 12 months to assess caller appraisal and quit rates.

Setting: The Australian Quitline service, in the context of a nationally coordinated, major anti tobacco campaign.

Results: In a one year period from June 1997, 3.6\% of adult Australian smokers called the Quitline. Weekly call volume was strongly related to TARPs and increased further when an advertisement specifically promoting the Quitline was broadcast. Calls involving requests for counselling, as opposed to brief calls to request quit materials, were more likely with lower TARPs. Of the cohort who were smoking at baseline, $28 \%$ reported they had quit smoking at a one year follow up and $5 \%$ had been quit for an entire year.

Conclusions: In the context of a national mass media campaign, this study illustrates that it is possible to bring together differing State based services to provide an accessible, acceptable, and effective quit smoking service.
\end{abstract}

Q uitlines or smokers' helplines are identified as a valuable component of any large scale tobacco control programme. ${ }^{1}$ They provide relevant, accessible, flexible, and affordable methods of quitting smoking for the population..$^{1-5}$ Reactive telephone services, such as helplines, have been appraised as effective cessation aids in their own right, and are utilised as an adjuvant to pharmacotherapy and an important referral point for physicians and other health professionals. ${ }^{167}$

The response to helplines depends directly on promotion to advertise their availability to smokers. ${ }^{8-9}$ Increasingly, helpline services are being used to provide a first line source of accessible quit smoking assistance within the context of mass media anti-smoking campaigns. ${ }^{35}{ }^{10-12}$ The aim of these services is to provide assistance to as many smokers as possible, within the population of smokers. Within this context, the first objective must be to provide minimal forms of assistance, rather than more intensive counselling. ${ }^{13-14}$ This means that the service must be organised in a way that permits prospective callers to access the service when they wish to, even during periods of intensive media advertising, when call volume may be very high.

Once the helpline has established its capacity to provide a basic cessation intervention to all of its callers, even at times of high demand, then the issue of value adding, or more intensive interventions, can be addressed. Studies have demonstrated the additional benefits to smokers receiving more intensive interventions (such as counselling), delivered via quitlines. ${ }^{715}$

The challenge of implementing a telephone helpline service that has sufficient flexibility to meet this requirement, while still providing assistance appropriate for the different needs of callers, is one that is presently the subject of considerable interest and debate. Helpline services, which have as their target the entire population of smokers, should be judged not only by the reported quit rates of callers to the service, but by the number of calls able to be generated by the advertising and the capacity of the system to answer calls when they are placed. The value of these services is also related to their cost effectiveness.
This paper presents a case study of the Australian telephone Quitline service, which was integrated to form a national service as part of the Australian National Tobacco Campaign (NTC). The aims of the study were to document number and type of calls to the Quitline service over the period of the first year of the campaign; to monitor the capacity of the system to deal with fluctuating call volume; to determine the characteristics of callers; and to assess callers' appraisal of the service and short and longer term self reported change in smoking behaviour.

\section{METHODS}

\section{Establishing the Australian national Quitline service}

Beginning in June 1997, Australia mounted its first nationally coordinated anti-smoking campaign, with three television advertisements that promoted the message "Every cigarette is doing you damage". The advertisements graphically illustrated the damage smoking inflicts upon lung tissue, arteries, and genes in lung cells. ${ }^{16-17}$ From April 1998, two additional advertisements were broadcast-one graphic advertisement that focused upon smoking and strokes, and one that solely promoted the idea of calling the Quitline. ${ }^{17}$ The last advertisement showed a male caller ringing the Quitline and demonstrated the types of assistance available, demystifying the Quitline in an effort to reduce one potential barrier to calling. ${ }^{14}$ All five advertisements were tagged at the end with the Quitline number, which appeared for approximately one and a half seconds. Television advertising was broadcast intensively for the first four weeks (June 1997), followed by a period of high and low intensity advertising in alternate weeks, tailing off into December 1997. The advertising peaked again at New Year and in April 1998 when the new stroke and Quitline advertisements were broadcast.

Abbreviations: TARPs, television target audience rating points; NTC, National Tobacco Campaign; ADIS, Alcohol and Drug Information Services 
Although television advertising was the dominant medium for the campaign, a range of other media and promotional activities were employed to extend the reach of the key advertising messages. Although most smokers quit without formal assistance, many do seek help and, therefore, additional elements of the campaign involved the distribution of quit smoking resources and the provision of professional cessation services, principally the telephone Quitline. For example, letters and order forms for quit smoking materials were sent to all Australian general practitioners and pharmacists to encourage their participation in providing assistance to smokers to quit.

The Quitline service could be accessed by the public by dialling a single telephone number from anywhere in the country for the cost of a local call in metropolitan areas and for no charge to callers outside of metropolitan areas. The calls were automatically answered in the State or Territory from which they originated. For a range of historical reasons each jurisdiction had slightly different arrangements for answering calls. For answering all calls in the first instance, five (out of eight) jurisdictions subscribed to a telecentre telephone answering service, one answered all calls in its own Quit campaign office, one used counsellors employed by Alcohol and Drug Information Services (ADIS), and one State used a recorded interactive voice mail system. Prior to the launch of the NTC, a cessation services committee formed, comprising Quitline representatives from every Australian State and Territory, in order to develop a minimum service protocol that could be applied in all jurisdictions

Despite different structural arrangements, it was agreed that all calls would be initially answered with the greeting "Hello, this is the Quitline. Would you like me to send you a Quit book?" If callers agreed, their contact details were collected and they were told the book would reach them in the next few days. Callers who spontaneously expressed the wish to talk further about their smoking, were then either transferred to a counsellor, either in the State's Quit campaign office or to a counsellor at ADIS, depending on each jurisdiction's particular set up. Callers who initially accessed an ADIS counsellor were greeted in the same manner, and if they expressed the wish to talk further about their smoking, the counsellor went ahead with further discussion. After the first four weeks of the campaign, when it was established that call volume was manageable, counselling was offered proactively. After being offered a Quit book all callers were asked, "Would you like to talk with someone now about your smoking?" The only exception to this protocol was in the one State using an interactive voice mail system. These callers could either select an option to receive a Quit book, requiring them to leave their name and address on the message, and/or select an option that immediately transferred them to speak in person with a counsellor about their smoking. Although standardised records of call duration were not kept, it was usual for most calls put through to counsellors to last between 5 and 15 minutes.

The Quit book was a self help resource that had been developed and pre-tested with smokers and then produced in a standard fashion for use by all States (an electronic copy of this resource is available from: www.health.gov.au/pubhlth/ quitnow/quitbook/). It contained information pertinent to smokers at different stages of change. Specific information and pictures were included about the effects of smoking on health and benefits of quitting, getting ready to quit and setting a quit date, and advice about dealing with withdrawal symptoms, coping with urges to smoke, and dealing with lapses back to smoking. Other self help written resources were available for specific issues, on request.

\section{Monitoring of call volume}

The telephone carrier company, Telstra, was commissioned to provide a weekly monitor of calls made to the Quitline number, the number answered, and the number that rang out (unanswered) or received an engaged signal. Monitoring of the proportion of calls that were transferred for counselling was also undertaken by the telecentre service in those States using this service.

Records were also kept of the amount of television advertising associated with the campaign. Television advertising was measured in target audience rating points (TARPs). ${ }^{17}$ TARPs are the multiples of each $1 \%$ of the target audience (aged 18-40 years) who were exposed to the campaign, on the basis of the placement of television advertisements. TARPs can be increased by running the advertisement more frequently or in higher rating television programmes, or both. Because television advertising was the most dominant medium for this campaign, TARPs represent the most direct index of paid campaign activity. TARPs were tabulated for each week of the first year of the campaign.

\section{Follow up of Quitline callers}

Over a two day period in the third week of the campaign, all callers to the Quitline who met specified criteria were asked to consent to a call back for the purpose of providing feedback on the Quitline service and resources. This methodology was used to limit potential biases due to changing caller composition or changes to Quitline service protocols over the course of the campaign. To be eligible, callers had to be calling for themselves, aged 18 years or older, and had to be current smokers or recent quitters (defined as having quit within the past week). Eligible callers who consented to the call back were then asked for their name, address, telephone number, and preferred time to receive their call back. A note was then made as to whether they had been sent a Quit book, other resources, or no resources; the time of the index call; and whether the person had received counselling, defined as being transferred by the telecentre to a counsellor, or in other States not using a telecentre, having gone on to discuss their smoking with a counsellor after determination of whether a Quit book was required.

Follow up interviews were conducted 3 weeks, 6 months, and 12 months after the time of the initial call to the Quitline. The 3 week survey sought information about caller appraisal of the Quitline service, receipt and appraisal of the Quit book, any early quit attempts, and cessation. Where callers indicated they had not received their Quit book, address details were checked and an additional copy was posted. The 6 and 12 month follow up surveys asked about quit attempts and reported cessation, with the 12 month follow up additionally asking about re-contact with the Quitline over the period of the year since they first called. Up to six attempts were made to contact callers at follow up.

To assess appraisal of the service, callers were asked whether they found the service convenient and helpful, and whether the information it provided was relevant to their needs. In addition, callers were asked whether they would recommend the Quitline service to friends. Similar questions were asked about the Quit book.

At the 6 and 12 month follow up points, callers were asked whether they had attempted to quit smoking since the last contact, if they were smoking, the usual number of cigarettes smoked per day, and whether they had made any other changes to their smoking since calling the Quitline, such as delaying smoking in certain situations. Point prevalence cessation was defined as self reported quitting with no criteria about the time since quit, whereas period prevalence cessation was defined as quit at the time of follow up, as well as having been quit since the previous follow up and no cigarettes smoked in between. Quit rates are presented as a proportion of those able to be contacted at each survey, rather than the more conservative estimates generated using all callers at baseline as the denominator. Comparison of baseline differences, 


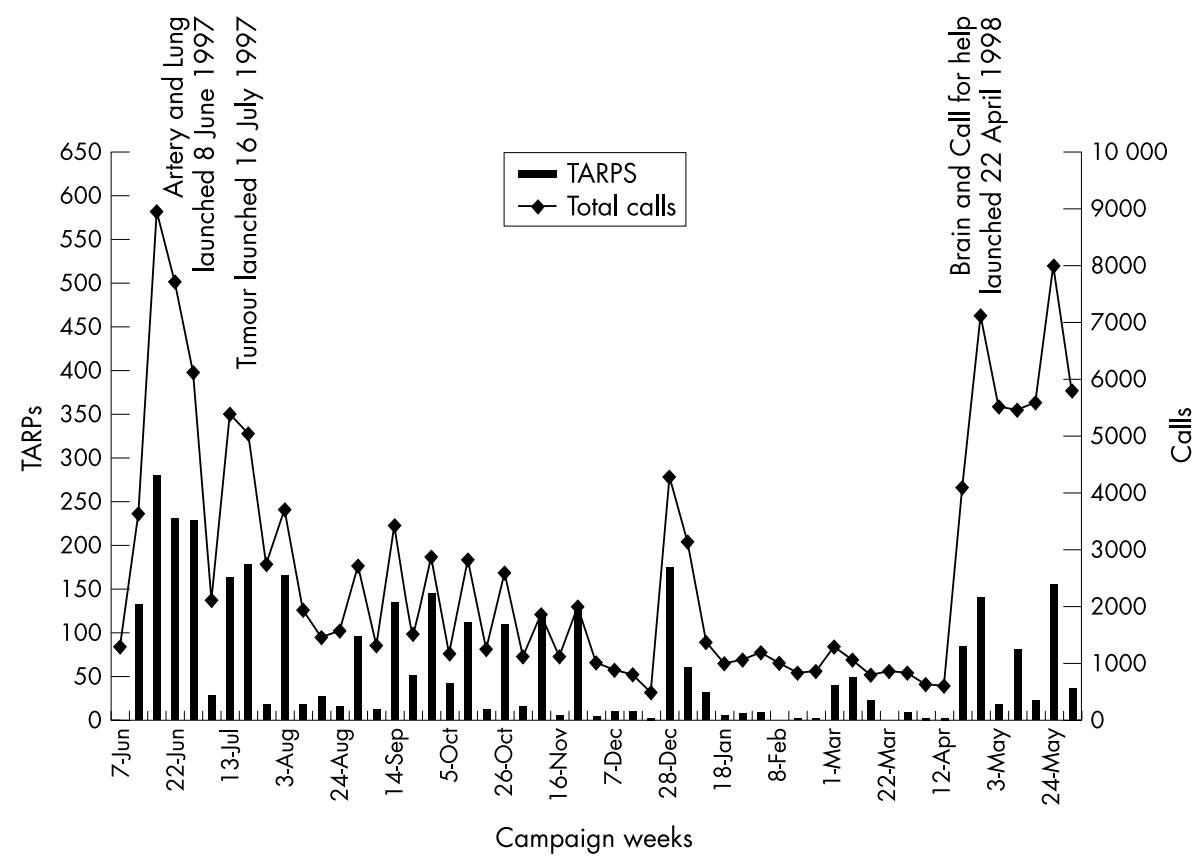

Figure 1 Relationship between television target audience rating points (TARPs) and calls to the Quitline, June 1997 to May 1998

Campaign weeks

between those able and not able to be contacted at each follow up, were conducted and are presented to support this approach

\section{RESULTS}

Trends in weekly call volume

Overall, in the first year of the campaign, 144500 calls were placed. It was estimated that $8 \%$ of calls were from nonsmokers ${ }^{10}$ and, from the follow up sample, $7 \%$ were repeat contacts from smokers who had called previously. Discounting these additional calls gave a figure of 123600 callers, representing 3.6\% of Australian smokers aged $\geqslant 18$ years. ${ }^{18}$

Fig 1 plots the weekly Quitline call volume data against the national TARPs for the first year of the campaign. During the first 9 months, three television advertisements depicting the damage smoking does to the body were aired (artery, lung, and tumour), and over this period Quitline call volume was strongly correlated with TARPs $(r=0.93 ; p<0.001)$. In the last 2 months, when a new advertisement depicting bodily damage (brain) was broadcast in tandem with a new advertisement that specifically promoted the Quitline (call for help), the relationship between TARPs and Quitline call volume changed, such that call volume was greater than that explained by TARPs alone. Regression analyses demonstrated a clear linear relationship between volume of TARPs and volume of calls to the Quitline, and a significant increase in call volume after the introduction of the call for help advertisement $(\mathrm{f}(2,49)=183.2 ; \mathrm{p}<0.001)$. Responses for individual State and Territories are available elsewhere. ${ }^{19}$

\section{Weekly Quitline call volume, by weekly TARPs}

During the first year of the campaign 132800 calls were answered, representing $92 \%$ of calls made. There was variation in weekly answering rates, ranging from $79 \%$ to $98 \%$. Answering rates were significantly lower during weeks of intensive television advertising when TARPs were 100 or greater $(90.4 \%)$, than weeks when TARPs were lower $(93.0 \%)$ $\left(\chi^{2}=294.8, \mathrm{df}=1, \mathrm{p}<0.0001\right)$.

Using the subset of data for the States of South Australia and Victoria, which used the telecentre continuously throughout the campaign, an average of $28 \%$ of calls were transferred each week for counselling (range 23-36\%) for the first four weeks of the campaign. After week four, with the introduction of a proactive invitation to talk to a counsellor, the average increased to $51 \%$ in the weeks thereafter (range 32-68\%). Excluding the first four weeks, when there was no proactive offer to transfer to a counsellor, the percentage of calls transferred for counselling was significantly lower in weeks when TARPs were 100 or more $(43.5 \%)$, than during the weeks TARPs were less than $100(54.9 \%)(f(1,46)=25.7, p<0.001)$.

\section{Follow up of Quitline callers}

Overall, there were 1831 callers eligible to take part over the two day period of recruitment, of whom 1568 (86\%) consented to take part. Over the period of recruitment, a greater number of calls were received in five of the States than was required for the purposes of the call back survey (based on the necessary final sample and anticipated attrition rates). Therefore, the sample was capped in each of these States and the required number of call back phone numbers was selected by a random process, yielding 1090 eligible and consenting callers.

There were 920 callers (84\%) successfully contacted for interview at the 3 week follow up, 676 (62\%) at 6 months, and $494(45 \%)$ at 12 months. Cumulative reasons for non-response at the 12 month point included: caller moved and not able to be traced (29\%); no answer after six attempts (17\%); caller away for duration of survey period ( $1 \%$ ); and ineligible for interview due to younger age or language barrier $(<1 \%)$. Only $7 \%$ of respondents actively refused to be interviewed. At each time point there were no significant differences between callers able to be contacted and those who were not with respect to sex, level of educational attainment, region of residence, quit attempt history at baseline, baseline smoking status, and mean daily cigarette consumption, or whether the caller selected a brief or longer counselling call at baseline. However, at the 12 month follow up those lost to follow up were significantly younger than participating respondents $(\mathrm{f}(1,906)=49.59 ; \mathrm{p}<0.001)$.

Of the 920 callers successfully recontacted 3 weeks after their index call, $79 \%$ were aged $18-40$ years, being the primary target age group for the campaign. Overall, 53\% were female and $58 \%$ reported high school education but no further study. According to their telephone numbers, 56\% of callers were from metropolitan areas, 37\% from country areas of Australia, and $7 \%$ were from mobile phones. When asked about their smoking behaviour at the time of the index call, $97 \%$ were 
Table 1 Quit rates and change among continuing smokers at follow up

\begin{tabular}{lllll}
\hline & Baseline & 3 weeks & 6 months & 12 months \\
\hline Number of callers & 920 & 920 & 676 & 494 \\
Point prevalence (quit at time of index call/follow up) & $3 \%$ & $19 \%$ & $24 \%$ & $29 \%$ \\
Period prevalence (quit for $\geqslant 6$ months) & na & na & $8 \%$ & $14 \%$ \\
Period prevalence (quit for 12 months) & na & na & na & $6 \%$ \\
Number of continuing smokers & & & & \\
Mean daily cigarettes (sd) & 288 & 743 & 517 & 351 \\
Positive change in smoking pattern & $22(13)$ & $15(11)$ & $18(11)$ & $18(11)$ \\
Tried to quit since index call & na & $32 \%$ & $6 \%$ & $9 \%$ \\
& na & $37 \%$ & $74 \%$ & $81 \%$ \\
\hline
\end{tabular}

smokers and 3\% had quit in the week prior to calling the Quitline. Among the smokers, mean cigarette consumption was 22.2 cigarettes per day $(\mathrm{sd}=12.5)$, with $44 \%$ smoking 25 or more cigarettes per day. Of the smokers, $50 \%$ reported having tried to quit in the past year. When they called the Quitline, $80 \%$ of callers reported they had a brief interaction with the person who answered the phone and the remaining $20 \%$ said they had spoken with a counsellor at some length about quitting.

At the 3 week follow up, callers favourably appraised the service they received from the Quitline, with $98 \%$ reporting it to be somewhat or very convenient, $86 \%$ to be somewhat or very helpful, and $82 \%$ to be somewhat or very relevant. Overall, $61 \%$ of callers said they would definitely recommend the service to friends.

In relation to the Quit book, $86 \%$ of callers reported they had received their Quit book and of these, $73 \%$ had received it within 5 working days. The Quit book was rated very favourably, with all callers reporting it to be easy to understand, $97 \%$ judging it to be very or somewhat relevant to them, and $94 \%$ somewhat or very helpful. Furthermore, $92 \%$ said they would definitely or probably recommend it to friends.

Table 1 shows that at the 12 month follow up 29\% of callers reported having quit smoking (point prevalence), with $14 \%$ having quit for 6 months or more and $6 \%$ for 12 months. Excluding those who were recent quitters at baseline from the panel and considering baseline smokers only, quit rates at the 12 month point were $28 \%$ for point prevalence, $14 \%$ for 6 month period prevalence, and 5\% for 12 month period prevalence. Therefore, quit rates mainly reflect new cessation among smokers at baseline, and are not simply a function of the fact that the original sample contained recent quitters who maintained cessation. A more conservative way to analyse these data would be to employ intention to treat analyses. If, at the 12 month follow up, all of those lost to follow up were (almost certainly wrongly) assumed to be smoking, the point prevalence would be $13 \%$, while $6 \%$ would have been quit for a period of at least 6 months and only 3\% would have been quit for a period of 12 months.

Table 1 also shows that among those who continued to smoke, daily cigarette consumption reduced significantly at the 3 week follow up, but had risen again by the 6 and 12 month follow ups, albeit not returning to baseline levels. Making positive changes to one's smoking habit, such as changing where and when a cigarette was smoked and reducing consumption, was common at the 3 week follow up but there was little new change after this time. Attempts to quit smoking occurred disproportionately in the first 3 weeks after the index call, although by the 12 month follow up $80 \%$ of callers had made a quit attempt.

In the year following their index call, 31\% of the 494 in the sample reported that they considered calling back the Quitline. Overall, 7\% of the sample did call back and in total, $3 \%$ called back on more than one occasion. Of the 35 people who had called again, reasons for doing so mainly related to seeking help in making another quit attempt (40\%) or help to stay quit $(20 \%)$.

\section{DISCUSSION}

The experience of developing and implementing a national telephone Quitline service to support the NTC showed that it was possible to bring together disparate State based models for service provision and integrate them in a standard way to provide an accessible, acceptable, and effective quit smoking service.

The prime purpose of media advertising was to make smokers seriously think about quitting and ultimately to quit. The Quitline was promoted within the campaign advertising as an inexpensive and easily accessible service for those smokers who did wish to avail themselves of advice. It would not be expected, or desirable, for all smokers to require assistance from services such as the Quitline. However, in the context of a mass media campaign, calls to the Quitline can be construed as the "tip of an iceberg" of quitting activity in the population.

Over the first year of the NTC, nearly one in 25 adult smokers called the Quitline and call volume was strongly related to TARPs. It is worth noting that the tagline promoting the Quitline number appeared at the end of each of the "damage" advertisements for less than 2 seconds, included no specific call to action ("ring now . ."), and no offer ("ring now and we'll provide counselling . ..."). ${ }^{20}$ If these direct marketing principles had been adhered to, it is probable that call volume would have been even higher. There were valuable lessons learned with respect to media scheduling in generating Quitline calls. Although investment in television advertising was related to call volume in a fairly predictable manner, the addition of an advertisement that specifically promoted the Quitline increased calls over and above that predicted.

Another direct marketing principle is that of "push/pull" where the first advertisement or first part of an advertisement motivates people to think about change, and the second part provides them with a way of achieving it, or accessing the product. This is much the same thing as pairing a stimulus (an advertisement designed to make people think about the need to quit smoking) with a cue to action (quitline advertisement, promoting a service for helping them to do so). When this principle was used in the April 1998 component of the campaign, where the brain advertisement was run in tandem with the Quitline advertisement, a vast increase in the number of calls resulted, given the TARPs invested. Subsequent national monitoring of the Quitline service has demonstrated that this volume and pattern of calls has persisted over subsequent years of the NTC.

Over the first year of the campaign $92 \%$ of callers had their call answered. This was an acceptable answering rate in the context of an unprecedented and highly fluctuating call volume. The slight but significant drop in the proportion of calls answered during times of higher media activity serves as a reminder that first it is crucial to be able to meet the demand that is created for the service at a basic level before adding value with extended counselling, which may reduce population access to the basic service. The variation over time in the percentage of callers accessing counselling deserves comment. 
Firstly, it was noted that the percentage of callers accessing counselling doubled once a more proactive offer was made, suggesting that many smokers were unclear as to what type of advice or help they expect (or perhaps require) from such a service in order to try to quit. Secondly, it was found that the percentage of callers requesting counselling tended to be higher in the weeks when the advertising investment was lower, as measured by TARPs, than in the weeks when it was higher. This may be partly related to the call operators having more time available to talk with callers during the weeks when the television advertisements were not being aired, because the call volume was lower, although this is less likely to have been the case for call centre staff who transferred callers requesting counselling. Another factor accounting for this pattern may be that people who call when the television advertising is not being broadcast must have written down the number for future reference, or obtained it from another source (for example general practitioners or other health services). Therefore, they may be making a more deliberate and considered call for assistance than callers who call spontaneously after seeing the number on television. These factors that influence call volume and type are important to consider in relation to staffing of telephone helplines.

The timely dispatch and receipt of Quit books is another important indicator of the ability of the service to meet callers' needs in terms of providing help for their immediate interest in quitting, and is important in assessing the service's capacity to respond to demand and maintain credibility. Measuring the rate and speed of receipt of the books highlighted a mailing system problem in one jurisdiction, which was quickly remedied. The absence of monitoring would have delayed this identification and resolution.

Rates of self reported point prevalence were higher during the 12 month follow up survey (29\%) than during the 6 month follow up survey $(24 \%)$. It is important to note that the 12 month follow up occurred in the month following the launch of the new damage related and Quitline television advertisements. This advertising may have prompted new quitting activity among the panel of callers to the Quitline and so account for the high point prevalence quit rates at 12 months. However, the proportion of callers who had sustained a quit attempt for the previous 6 months was also higher at the 12 month follow up (14\%) than at the 6 month follow up (8\%), so it is unlikely that the television advertising in April fully accounts for the increased quit rates at 12 months.

It is difficult in the context of evaluating a service like this to obtain a control or comparison group. When operating a reactive helpline that is promoted through anti-smoking advertising, all smokers are potentially exposed to the advertising and self select as callers, thereby differentiating themselves in some way from those who do not call. It is often noted that callers to helplines or quitlines are likely to be highly motivated to quit, which could be expected to lead to better cessation outcomes than those observed in a population sample of smokers who do not use such a service. ${ }^{12}$ However, other important differences between those who call and those who do not call have been noted. Studies reporting on the characteristics of callers to the English Quitline and the Massachusetts Smoker's Quitline have reported that smokers accessing those services smoked more heavily and were more highly addicted than the smokers in those populations who did not call. ${ }^{41}$ Callers who self select into receiving counselling, or a Quit book only, are also by definition different from each other, and it was not possible to randomise callers to receive different kinds of help in the context of a campaign in the field. However, other studies suggest that most telephone counselling protocols are effective for smoking cessation. $^{722}$

Notwithstanding these cautions, the outcome results found in this study compare favourably with similar evaluation data. Platt and colleagues, ${ }^{5}$ reported 12 month follow up data from a panel of callers to a telephone quitline in Scotland, which was promoted as part of a mass media anti-smoking campaign. The volume of calls per capita was not dissimilar, with an estimated $5.9 \%$ of adult smokers contacting the Scottish Smokeline in its fifth year of operation, compared with $3.6 \%$ in this study. Point prevalence quit rates of $24 \%$ at 12 months were reported, and $8 \%$ of those who were quit at the 12 month follow up had been quit for at least $80 \%$ of the year. The comparable results from the Australian service were 29\% and $11 \%$, respectively. Many other jurisdictions in the United States, Europe, and the United Kingdom, as well as New Zealand, have also established smokers' helplines or quitlines and have reported achieving sound results (H Glasgow, personal communication, 2002). ${ }^{323}$ Most recently, researchers embedded a randomised controlled trial design into the ongoing Californian smoker's helpline, ${ }^{23}$ demonstrating better cessation outcomes for the treatment group (which received up to seven counselling sessions) than the control group (which received self help materials only). This study expands upon efficacy trials and evaluations without controls and demonstrates effectiveness of the service in a "real world" setting.

In order to increase the number of quitters through the Quitline service, one could implement strategies to increase call volume and/or one could add in additional intervention components to increase the quit rates of callers, such as a call back service ${ }^{715}$ and several Australian jurisdictions have since added this option to their service. However, the addition of more assistance options in an effort to increase the quit rates of callers should not be at the expense of making the basic service less accessible, by tying up telephone lines and decreasing the ability to maintain high answering rates, because the basic service achieves very acceptable rates of quitting smoking. For public health campaigns using helplines as an adjunct to assist cessation, these considerations are crucial to enable large numbers of callers to access the service.

Although it is known that most smokers prefer to quit without recourse to formal treatments or programmes, ${ }^{21}$ telephone helpline services can play an important role in assisting smokers to access information and advice about quitting. As this study has demonstrated, it is possible to bring together differing service models within the context of a mass media campaign to operate in a standard way, to deliver an accessible and effective quit smoking facility.

\section{ACKNOWLEDGEMENTS}

Funding for the study was provided by the Australian NTC and the Victorian Smoking and Health Programme. We wish to thank Tina Bleasdale and Pat Williams from Williams Media Audits for contributing data on TARPs.

\section{DISCLOSURE}

Melanie Wakefield is a senior editor of Tobacco Control. She was excluded from editor-reviewer correspondence and was not involved in the editorial decision making process for this manuscript.

\section{Authors' affiliations}

C L Miller, Tobacco Control Research and Evaluation, The Cancer Council South Australia, Australia

M Wakefield, Centre for Behavioural Research in Cancer, The Cancer

Council Victoria, Australia

L Roberts, National Heart Foundation of Australia, Australia

\section{REFERENCES}

1 Zhu S-H, Anderson CM, Johnson CE, et al. A centralised telephone service for tobacco cessation: the Californian experience. Tob Control 2000;9(Suppl II):ii48-55.

2 Lichtenstein E, Glasgow RE, Lando HA, et al. Telephone counseling for smoking cessation: rationales and meta-analytic review of evidence. Health Education Research 1996;11:243-57.

3 Owen L. Impact of a telephone helpline for smokers who called during a mass media campaign. Tob Control 2000;9:148-54.

4 Prout MN, Martibnez O, Ballas J, et al. Who uses the Smoker's Quitline in Massachusetts? Tob Control 2002;11 (Suppl II):ii74-5. 
5 Platt S, Tannahill A, Watson J, et al. Effectiveness of antismoking telephone helpline: follow up survey. BM 1997;314:1371.

6 Zhu S-H, Tedeschi G, Andersen CM, et al. Telephone counselling as an adjuvant treatment for nicotine replacement therapy in a "real world" setting. Prev Med 2000;31:357-63.

7 Zhu S-H, Stretch V, Balabanis M, et al. Telephone counseling for smoking cessation: effects of single session and multiple session intervention. J Cons Clin Psychol 1996;64:202-11.

8 Ossip-Klein DJ, Giovino GA, Megahed N, et al. Effects of a smokers' hotline: results of a 10-county self-help trial. J Cons Clin Psychol 1991;59:325-32.

9 Cummings KM, Sciandra R, Davis S, et al. Results of an anti-smoking media campaign utilizing the Cancer Information Service. J Natl Cancer Inst Monogr 1993;14:113-8.

10 McCabe P. Making the phone ring: promoting a Quitline. How to operate a telephone quitline service for tobacco cessation. Workshop sponsored by the California Department of Health Services and the American Cancer Society. San Diego, California: 28-29 August 1998.

11 Wilson N, Hodgen E, Mills J, et al. Events of 11 September 2001 significantly reduced calls to the New Zealand Quitline [Letter to the Editor]. Tob Control 2002;11:280.

12 Ratcliffe J, Cairns J, Platt S. Cost effectiveness of a mass media-led anti-smoking campaign in Scotland. Tob Control 1997;6:104-10

13 Zhu SH, Rosbrook B, Anderson C, et al. The demographics of help-seeking for smoking cessation in California and the role of the California Smokers' Helpline. Tob Control 1995;4:S9-15.

14 Wakefield $M$, Borland R. Saved by the bell: the role of telephone helpline services in the context of mass-media anti-smoking campaigns. Tob Control 2000;9: 117-9.
15 Borland R, Segan CJ, Livingston PM, et al. The effectiveness of callback counselling for smoking cessation: a randomised trial. Addiction 2001;96:881-9.

16 Hill D, Chapman S, Donovan R. The return of scare tactics. Tob Control 1998;7:5-8.

17 Hill D, Carroll T. Australia's National Tobacco Campaign. Tob Control 2003;12(Suppl II):ii9-14.

18 Australian Bureau of Statistics. National Health Survey 1995: Health Risk Factors, Australia. Catalogue number 4369.0. Canberra: Australian Bureau of Statistics, 1998.

19 Wakefield M, Miller C. Evaluation of the National Quitline Service. In: Australia's National Tobacco Campaign: evaluation report volume one. Canberra: Commonwealth Department of Health and Aged Care, 1999. www.health.gov.au/pubhlth/publicat/document/metadata/ tobccamp.htm

20 Williams $\mathbf{P}$, Bleasdale T. The relationship between campaign television activity and Quitline call data. In: Australia's National Tobacco Campaign: evaluation report volume one. Canberra: Commonwealth Department of Health and Aged Care, 1999.

21 Owen N, Davies M. Smokers' preferences for assistance with cessation. Prev Med 1990;19:424-31.

22 Orleans CT, Schoenbach VJ, Wagner EH, et al. Self-help quit smoking interventions: effects of self-help materials, social support instructions and telephone counseling. J Cons Clin Psychol 1991;59:439-48.

23 Zhu SH, Anderson CM, Tedeschi GJ, et al. Evidence of real-world effectiveness of a telephone quitline for smokers. N Engl J Med 2002;347:1106-9. 